\title{
CASE STUDY/OPINION
}

\section{Technological Explorations in the Move to Online Mathematics Support}

Calum Heraty, Department of Mathematics and Statistics, Maynooth University, Ireland.

Email: calum.heraty@mu.ie

Ciarán Mac an Bhaird, Department of Mathematics and Statistics, Maynooth University, Ireland.

Email: ciaran.macanbhaird@mu.ie

Aisling McGlinchey, Department of Mathematics and Statistics, Maynooth University, Ireland.

Email: aisling.mcglinchey@mu.ie

Peter Mulligan, Department of Mathematics and Statistics, Maynooth University, Ireland.

Email: peter.mulligan@mu.ie

Pádhraic O'Hanrahan, Department of Mathematics and Statistics, Maynooth University, Ireland.

Email: padhraic.ohanrahan@mu.ie

James O'Malley, Department of Mathematics and Statistics, Maynooth University, Ireland.

Email: james.omalley@mu.ie

Rachel O'Neill, Department of Mathematics and Statistics, Maynooth University, Ireland.

Email: rachel.oneill@mu.ie

Tara Vivash, Department of Mathematics and Statistics, Maynooth University, Ireland.

Email: tara.vivash@mu.ie

\section{Abstract}

Due to Covid-19, mathematics support at Maynooth University transitioned from in-person to online over a very short period in March 2020. This paper provides a brief description of the technologies used by six tutors to facilitate this move. We outline why the tutors picked these technologies and how they used them. We also consider the issues tutors wanted to resolve and reflect on the outcomes of their experiences.

Keywords: Online mathematics support, technology, resources.

\section{Introduction}

On Thursday $12^{\text {th }}$ of March 2020, due to Covid-19, the Irish Government announced the closure of Higher Education Institutions for face-to-face teaching. Due to a mid-term break the following week, this gave Maynooth University (MU) six working days before teaching restarted, exclusively in an online environment. As all staff and students at MU had access to Microsoft (MS) Teams, the Department of Mathematics and Statistics (Department) decided to support modules by getting Department and Mathematics Support Centre (MSC) tutors to respond to student queries via dropin sessions on MS Teams. Students could log on and ask tutors questions in real time.

Due to Covid-19 guidelines, tutors were required to work from home. With the short transition time to online teaching, tutors received some guidance but, in the main, the responsibility was theirs to provide the best support they could to students with the resources they had available. Prior to this, none of the tutors had used MS Teams, or most of the technologies outlined below.

Coincidentally, six MSC tutors were involved in ongoing tutor training. Lawson et al. (2019) identifies training as crucial for tutors of mathematics learning support (MLS) and approximately half of Higher 
Education Institutions (HEls) in Ireland and the UK with MLS provide such training. Grove et al. (2019) give a detailed description of the development of MLS tutor training in Ireland and the UK. Five of these tutors had several years of face-to-face tutoring experience and were on short-term contracts. The remaining tutor, Tara, was a postgraduate student in her first year of tutoring. One aspect of the tutor training involved the use of technology for the development of digital supports. When in-person MLS ceased, the six tutors were encouraged to use their experience of moving to online MLS as part of their digital training and to write a brief report.

In this paper, we use the individual tutor reports as a basis from which to outline the specific technologies they used, how they supported students, and finally, to reflect on the outcomes of their experience. While these were short-term interventions, developed quickly, in difficult circumstances, we hope that sharing an overview of their experiences will assist the MLS community with making informed decisions about the nature of online support they may provide.

\section{Examples}

\subsection{Wacom Tablet}

\section{Why this technology/support?}

Prior to the onset of Covid-19, the Department already had some 'One by Wacom' creative pen tablets (https://www.wacom.com/en-us/products/pen-tablets/one-by-wacom) available to staff. The tutor (James) had previously used the tablet to record screencasts and wanted to use it, in conjunction with a virtual whiteboard, so students could see him working through problems in real time.

\section{Technical details.}

The tablet (see Figure 1) comprises of a pen and a writing surface that connect to PCs or laptops via USB. The pen can be used to control the mouse by moving it over the drawing surface. This allows for accurate writing of text and mathematical notation on a computer screen. If the user installs the generic Microsoft graphics tablet driver, then the mapping from the writing surface to the computer screen is not a bijective one. This makes writing on the screen much more difficult, but the issue is easily resolved by installing the product specific driver from the Wacom website.

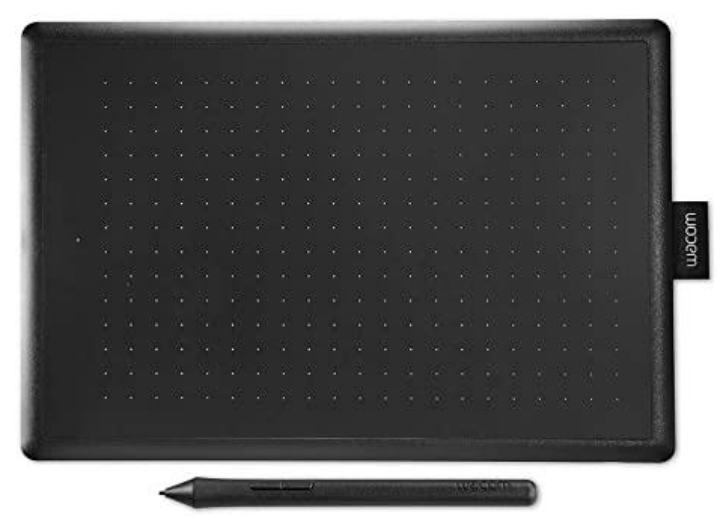

Figure 1: Wacom tablet and

pen 


\section{How the resource(s) were used to support students.}

James used the Wacom tablet along with the screen sharing feature of MS Teams during his online hours, approximately 19 each week. When students logged on, they would ask a question or provide pictures of work they had done. James would open the free MS Whiteboard app, share his screen with students and use the Wacom tablet and pen to facilitate clear and accurate writing (see Figure 2). The tablet was also used, along with screen casting software (Explain Everything), to produce 8 short videos to address recurring student problems during drop-in sessions.

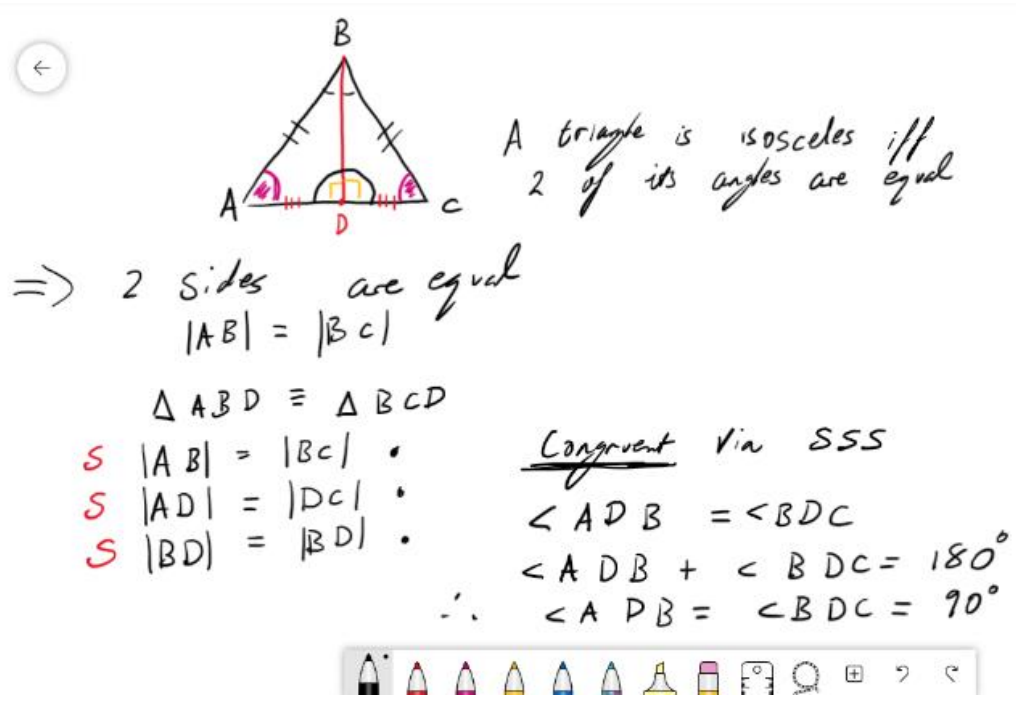

Figure 2. Example of an explanation on MS Whiteboard written via the Wacom tablet.

\section{Outcomes.}

James found the tablet to be intuitive and responsive. It felt like using a real pen and, after prolonged use, the user can utilise the pen without looking at the writing surface. There was no negative feedback from students in terms of the legibility of the text written using the tablet.

\subsection{OneNote}

\section{Why this technology/support?}

OneNote software is provided to both MU students and staff as part of MS Office 365. Calum used this software because of its functionality as a virtual whiteboard and Rachel used it to overcome the issue of not having a tablet, such as described in Section 2.1, to write mathematical text for students.

\section{Technical details (Calum).}

OneNote provides a blank canvas with utilities that trump the conventional pen and paper. It provides unlimited space both horizontally and vertically, expanding as you input material. You can easily zoom in and out, either to focus on smaller details or to quickly locate previous work. A customisable toolbar provided pens and highlighters in any choice of size and colour. It has a variety of shapes that can be used to create text boxes and add directional arrows. All these resources are clearly visible, with no clutter or excess. 
How the resource(s) were used to support students (Calum).

Calum used OneNote during 13 sessions each week, and after each session the notebook could be converted to pdf and shared with attendees. Calum found the multiple tabs or pages feature, as described by Rachel (below), very useful for supporting students. OneNote also contains a variety of features that support mathematics directly. Selecting equations using the Lasso tool, in conjunction with the Mathematics tool, opened a side panel which allows the performance of numerous tasks. This includes automatically solving simultaneous equations or differentiating, with detailed steps of the process. A convenient feature was the ability to convert equations to 2D graphs and paste them anywhere in the workbook. This made OneNote ideal for preparing notes and presenting examples.

\section{Outcomes (Calum).}

Overall, OneNote proved to be an extremely versatile virtual whiteboard. However, some features were problematic in the circumstances. Without a tablet and stylus, writing with a mouse was difficult and quite slow, especially with legibility being so important. The Mathematics tool had limited ability to predict which mathematics symbols your ink represents. Although you can change incorrect symbols individually, a significant amount of time is lost when trying to correct more complex equations. This reduces its practicality in a live session.

\section{Technical details (Rachel).}

Rachel had a laptop, touchscreen stylus and a Samsung Galaxy tablet. She wanted to screen-share while writing on a digital whiteboard, but writing clearly with a mouse is difficult. She preferred writing on the tablet with the stylus, however, the tablet would not screen-share. Since OneNote can be used in a browser or as an app the same OneNote document (Notebook) could be opened simultaneously on both devices. This was ideal in the circumstances, as Notebooks can be edited collaboratively in real time and can be used as a digital whiteboard. Thus, edits made on the tablet could be seen updating on the laptop and vice versa. This allowed Rachel to use her laptop to screen-share while writing on the tablet.

\section{How the resource(s) were used to support students (Rachel).}

Being able to screen-share with students while writing, allowed the tutor to provide assistance which resembled in-person support.-Notebooks have sections and tabs to organise content, and they support text, drawing, uploading of images, and the embedding of videos and documents like PDFs. A Notebook was created for each of the four modules supported, collating various resources such as practice sheets, lecture notes and statistical tables. Rachel found it particularly useful that drawing tools could write over uploaded pictures and documents. Rachel used OneNote during 15 sessions each week.

\section{Outcomes (Rachel).}

Writing on one device and screen-sharing on another was cumbersome and is not recommended. A graphics tablet (such as One by Wacom) would easily remedy this situation. However, students, using just their mobile phones, could use OneNote and a cheap stylus to create their own Notebook with the content that they wanted support with. Then, during any sort of audio call, the student could share this Notebook with the tutor who could annotate it in real time.

\subsection{Group work via MS Teams}

\section{Why this technology/support?}

The purpose was to try and replicate, in an online space, the group work environment that students would normally experience in the MSC. 


\section{Technical details.}

Similar to 'breakout rooms', MS Teams uses 'channels'. In any Team, you can create up to 30 channels, containing up to 250 members in total. In these channels users can easily screen-share, upload and share files, collaborate on the whiteboard and make video/voice calls with each other. However, unlike breakout rooms which are temporary, these channels are private and permanent, and only available to members of the channel.

\section{How the resource(s) were used to support students.}

Pádhraic used channels to provide support to 27 students taking a pre-degree mathematics course. Before each session, students were allocated into six separate channels (Group A, Group B, etc.), each channel contained 4-5 students. During the session, students would work on questions together by setting up a meeting in their channel. The group could message if they needed help, and Pádhraic would then join their meeting. He would also periodically jump around each channel to check on student progress.

\section{Outcomes.}

Some students reported that it could be intimidating to ask a question in class, and that the small group size of their channels created a more inviting atmosphere for engagement. Pádhraic found multiple groups difficult to support at the same time. Also, if a common issue arose across multiple groups, the only way to discuss it with the entire class would be to arrange a separate meeting for all the students.

\subsection{Videos}

\section{Why this technology/support?}

Two tutors identified recurring issues during their drop-in sessions and decided to develop short videos with a view to having reusable resources available for all their students. Aisling used a free screen recorder, Free Cam, downloaded from www.freescreenrecording.com which recorded calculations on Microsoft Paint using a Wacom tablet. Tara did not have a tablet and used MS PowerPoint to create her videos.

\section{Technical details (Aisling).}

Free Cam records both your screen and audio. Unlike other free screen recorders investigated, e.g. Icecream Screen Recorder or Debut Video Capture Software, it had unlimited recording time and recordings could be edited. The screen recorder can be any size required and positioned anywhere on the screen. The recordings can be paused and restarted repeatedly using keyboard shortcuts. When editing, segments of the video or audio from sections of the video and volume can be adjusted. However, it is not possible to add anything (audio or video clip) after recording is finished.

\section{How the resource(s) were used to support students (Aisling).}

Aisling identified common issues of notation and poor exposition during her first-year drop-in sessions and grading. In the Department, students can be deducted marks in their coursework and final exams for exposition. As the issues were module specific, Aisling decided to create three bespoke videos, each under 15 minutes in duration, using Free Cam. One featured the correct use of 'equal to' and 'implies', and the other two on correct notation when answering integration and probability questions. These were placed on the first-year mathematics workshop Moodle pages. 


\section{Outcomes (Aisling).}

Aisling identified the benefits of being able to make a video with examples and detailed explanations to help students if they were having issues or struggling with a topic in mathematics. She identified the videos as reusable learning objects which could be available for any students struggling with the same topic.

\section{Technical details (Tara).}

For MS Powerpoint, mathematical notation was created on each slide (Insert Symbol $\rightarrow$ Equation $\rightarrow$ Ink Equation). The 'Record Slide Show' option was used to record audio inserted over each slide individually. The 'Appear' option allowed expressions to be revealed using the click of a mouse, and a pointer to hover over any part of the slide required. The file must be saved as a .pps (PowerPoint show) in order for it to automatically open as a show, however, for full functionality, the file must be opened in PowerPoint rather than PowerPoint online. To resolve this issue, a video can be created using PowerPoint (File $\rightarrow$ Export $\rightarrow$ Export to Video).

\section{How the resource(s) were used to support students (Tara).}

Tara discovered, towards the end of the second semester module, that first-year students were struggling to identify which method to use when presented with an integral. Due to the specific structure of the module, she could not find suitable materials online. She decided to create a video with a flowchart on identifying appropriate integration methods depending on the problem, and one video of worked examples to reinforce the use of the flowchart. Tara also used MS Forms to develop a quiz for students which allowed them to answer integral questions and receive an indication of those they got wrong along with correct answers. All materials were placed on the firstyear mathematics workshop Moodle pages.

\section{Outcomes (Tara).}

Tara found using PowerPoint to create videos a mixed experience. She had no tablet, and inserting mathematical notation was cumbersome but easier than writing with a mouse. She could prepare the slides in advance of recording the audio, and edit the audio slide by slide to fix any errors, rather than redoing the whole video. Unfortunately, she could not access the 'Insert Form' option to embed the quiz in PowerPoint. This would have allowed her to give clues to students if they had answered questions incorrectly. She felt that having the quiz separately on MS forms made it less interactive.

\section{Reflections and Future Work}

To reflect on the tutors' experiences and consider the implications for future online MLS at MU, the authors held a meeting in June 2020. This online meeting was also attended by Cormac Breen (Technological University, Dublin) and Michael Grove (University of Birmingham). They had received the six case studies in advance and their role was to give an external perspective to the discussion.

The sudden change from face-to-face teaching and the technology available to the tutors was identified as the largest influence on the level and type of online MLS provided. While student engagement with these and other MLS services were low, the tutors observed that once students engaged, they tended to return. This type of engagement with online MLS was commonplace in HE during this period (Hodds, 2020). However, the six trials impacted on Department practices. The trial of the Wacom tablet was positively received, and the Department ordered tablets for all tutors and many lecturers to use for teaching and support during the 2020-21 academic year. The functionality of OneNote proved very popular and most tutors are now using it as their virtual whiteboard of choice. High student engagement with channels in MS Teams, when compared with online drop-in sessions led the MSC to offer study group support through channels for all undergraduates in 2020-21. 
In terms of making resources for students, the development of practice and assignment quizzes on Moodle has been given priority by the Department for 2020-21. However, the MSC still creates screencasts for first-year workshops (while in-person MLS is not possible) but based on the tutors' experiences above, sections 2.1 and 2.4, the software 'Explain Everything', which is not free, is being used. Explain Everything has additional editing features, such as the ability to edit pen strokes if the voiceover is correct. This saves valuable time when recording these lessons. Our general approach to videos needs to be assessed carefully, and is being reviewed on and ongoing basis to consider accessibility factors and avoiding the replication of existing suitable resources.

The tutors highlighted that the two-way interaction between student and tutor, which is prevalent in an in-person MSC, needs to be replicated online. They commented on the danger of static teaching, with little or no input from students. Multiple paths to addressing this issue were suggested. Tutors found that microphones rather than text chat options seemed to get more students involved during live sessions, and that students should be encouraged to prompt the tutor's next steps.. The tutors also commented that they could not see what students were writing and that an interactive screen that both tutor and student could write on simultaneously would be desirable. For students that do not have access to a tablet, a cheap soft tipped stylus to write on their phone, as suggested by Rachel, may also work. The tutors also suggested asking students to send PDFs of their attempts which tutors could annotate or address in real-time. Regular use of online quizzes might also help students recognise that they need assistance. One tutor recommended https://itempool.com/ and the YouTube channel of 3Blue1Brown as a good place to start investigating interactive online teaching practices and banks of questions.

Tutors reflected on the challenge of determining student body language and facial expressions in an online setting. During in-person support, tutors can pick up on non-verbal cues regardless of what students might say. The tutors also realised how much they relied on their own gestures, facial expressions etc. when explaining in-person, and that they had to bear that in mind when explaining via audio, writing on screen or recording a video.

The tutors recognised the potential benefits of online support. For example, during in-person support, help is often 1-1 and a tutor may have to repeat a similar explanation several times to different students. Online drop-in tended to have more than one student attending, so one explanation could suffice and even promote discussion amongst students. The tutors also saw the merits of sharing existing videos, where possible, or developing short tailored videos to resolve recurring issues that students were encountering.

From the perspective of the MSC Director, the second author, these six case studies combined two very important features of MLS: ongoing tutor training (Lawson et al., 2019); and the exploration of online MLS, which a 2018 survey (Mac an Bhaird et al., 2020) suggested was not being used to its full potential. While these explorations of technologies and developments of online MLS supports were carried out over a very short period of time, they did have a significant influence on practices in the Department at MU. The case study reports will be available in full from the Teaching and Learning Mathematics Online website [http://talmo.uk/index.html] We hope that sharing this overview of the tutors' initial experiences will be of benefit to the wider MLS community. 


\section{Acknowledgements}

The authors thank Cormac Breen and Michael Grove for their assistance with the tutor meeting. The ongoing tutor training was part of the Developing Opportunities for Mathematics Educators (DOME) project http://www.imlsn.ie/images/Tutor Development/DOME Project.pdf. The tutor positions of James O'Malley and Rachel O'Neill were funded by the Higher Education Authority (https://hea.ie/).

\section{References}

Grove, M. J., Mac an Bhaird, C., \& O'Sullivan, C., 2019. Professional development opportunities for tutors of mathematics learning support. MSOR Connections, 14(3), pp. 4-15. https://journals.gre.ac.uk/index.php/msor/article/view/1021 [Accessed 13 November 2020].

Hodds, M., 2020. A report into the changes in Mathematics and Statistics support practices due to Covid-19. sigma Coventry: Coventry University. Available via http://www.sigma-network.ac.uk/wpcontent/uploads/2020/07/Report-into-the-changes-in-Maths-and-Stats-Support-practice-duringCovid-19.pdf [Accessed 13 November 2020].

Lawson, D., Grove, M. \& Croft, T., 2019. The evolution of mathematics support: a literature review. International Journal of Mathematical Education in Science and Technology, https://doi.org/10.1080/0020739X.2019.1662120 [Accessed 13 November 2020].

Mac an Bhaird, C., Mulligan, P., \& O'Malley, J., 2020. Mathematics Support Centres' Online Presence: Provision in Ireland and the UK in 2018. Teaching Mathematics and its Applications, https://doi.org/10.1093/teamat/hraa010 [Accessed 13 November 2020]. 